\title{
Kilometer-Long Ordered Nanophotonic Devices by Preform-to-Fiber Fabrication
}

\author{
Mehmet Bayindir, Ayman F. Abouraddy, Ofer Shapira, Jeff Viens, Dursen Saygin-Hinczewski, \\ Fabien Sorin, Jerimy Arnold, John D. Joannopoulos, and Yoel Fink
}

(Invited Paper)

\begin{abstract}
A preform-to-fiber approach to the fabrication of functional fiber-based devices by thermal drawing in the viscous state is presented. A macroscopic preform rod containing metallic, semiconducting, and insulating constituents in a variety of geometries and close contact produces kilometer-long novel nanostructured fibers and fiber devices. We first review the material selection criteria and then describe metal-semiconductor-metal photosensitive and thermally sensitive fibers. These flexible, lightweight, and low-cost functional fibers may pave the way for new types of fiber sensors, such as thermal sensing fabrics, artificial skin, and largearea optoelectronic screens. Next, the preform-to-fiber approach is used to fabricate spectrally tunable photodetectors that integrate a photosensitive core and a nanostructured photonic crystal structure containing a resonant cavity. An integrated, self-monitoring optical-transmission waveguide is then described that incorporates optical transport and thermal monitoring. This fiber allows one to predict power-transmission failure, which is of paramount importance if high-power optical transmission lines are to be operated safely and reliably in medical, industrial and defense applications. A hybrid electron-photon fiber consisting of a hollow core (for optical transport by means of a photonic bandgap) and metallic wires (for electron transport) is described that may be used for transporting atoms and molecules by radiation pressure. Finally, a solid microstructured fiber fabricated with a highly nonlinear chalcogenide glass enables the generation of supercontinuum light at near-infrared wavelengths.
\end{abstract}

Index Terms-Amorphous semiconductor, chalcogenide glass, integrated fiber, microstructured fiber, optical fiber, photonic bandgap, self-phase modulation, semiconducting nanowire, supercontinuum generation.

Manuscript received December 15, 2005; revised July 6, 2006. This work was supported in part by the Defense Advanced Research Projects Agency, in part by the Army Research Office, in part by the Office of Naval Research, in part by the U.S. Department of Energy, in part by the Institute for Soldier Nanotechnologies, and in part by the MRSEC Program of the National Science Foundation.

M. Bayindir was with the Research Laboratory of Electronics, Massachusetts Institute of Technology, Cambridge, MA 02139 USA. He is now with the Department of Physics, Bilkent University, Ankara 06800, Turkey (e-mail: mb@bilkent.edu.tr).

A. F. Abouraddy, O. Shapira, and J. Arnold are with the Research Laboratory of Electronics, Massachusetts Institute of Technology, Cambridge, MA 02139 USA (e-mail: raddy@mit.edu; ofers@mit.edu; jarnold@mit.edu).

J. Viens, F. Sorin, and Y. Fink are with the Department of Materials Science and Engineering, Massachusetts Institute of Technology, Cambridge, MA 02139 USA (e-mail: yoel@mit.edu).

D. Saygin-Hinczewski was with the Department of Materials Science and Engineering, Massachusetts Institute of Technology, Cambridge, MA 02139 USA. She is now with the Department of Physics, Istanbul Technical University, Istanbul 34469, Turkey.

J. D. Joannopoulos is with the Department of Physics, Massachusetts Institute of Technology, Cambridge, MA 02139 USA (e-mail: joannop@mit.edu).

Digital Object Identifier 10.1109/JSTQE.2006.882666

\section{INTRODUCTION}

$\mathbf{T}$ HE combination of insulating, semiconducting, and metallic elements in well-defined geometries and prescribed sizes, while forming intimate interfaces, is essential to the realization of practically all functional electronic, optoelectronic, and thermal devices [1], [2]. These devices are typically produced using a variety of elaborate wafer-based processes, which afford small features, but are restricted to planar geometries and limited coverage area. The use of this fabrication approach has been the cornerstone of the electronics revolution, but has had no impact on the optical fiber industry, which relies on a very different fabrication approach. Our goal here is to produce fibers that deliver electronic, optoelectronic, and thermal functionalities, which are maintained over extended lengths of a fiber. Our strategy in achieving this goal is to use the preform-based fiberdrawing technique that has proven to be simple, and yet to yield extended lengths of highly uniform fiber with well controlled geometries and excellent optical and thermal properties [3]-[11]. This new fabrication approach that we adopt thus relies on first preparing a large-scale macroscopic version of the required device, a preform, and then reducing it to the desired size through the process of thermal drawing. This process, however, places constraints on the materials that may be utilized. Nevertheless, a set of materials with widely disparate electrical, optical, and thermal properties has been identified and successfully incorporated into fiber-based devices.

In this paper, we discuss four distinct fibers and fiber-based devices in detail. In the first design illustrated in Fig. 1(a), a metal-semiconductor-metal (MSM) photodetecting core is surrounded with a multilayer resonant optical structure. Such an arrangement allows spectral filtering of the externally incident light before reaching the photodetecting core leading to narrowband photodetection [5]. In the second design depicted in Fig. 1(b), a hollow-core photonic bandgap (PBG) transmission line is surrounded with a thin temperature-sensitive semiconducting glass layer, which is contacted with electrodes to form independent heat-sensing devices. In this structure, the heat escaping from the core into the cladding, when high-power laser light is coupled into it, is detected in the form of an electrical current when radiation reaches the thin-film layer [8], [9]. In the third design, shown in Fig. 1(c), an omnidirectional reflecting multilayer structure is surrounded with metallic electrodes, which allows simultaneous transport of electrons and photons along the fiber [5]. The last design illustrated in Fig. 1(d), which is a solid nanostructured fiber consisting of a chalcogenide glass 
(a)

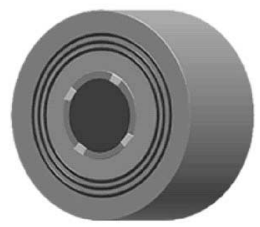

(c)

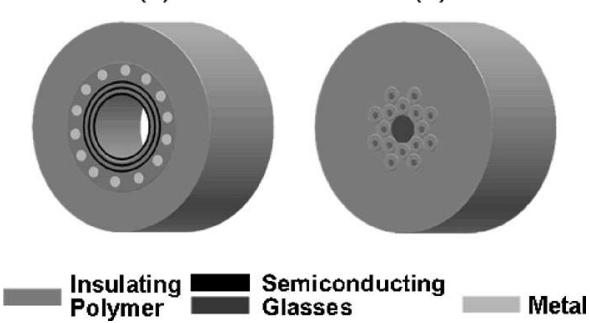

Fig. 1. Preform-to-fiber approach yields novel fibers and functional fiberbased devices. (a) A spectrally tunable fiber photodetector consisting of a photoconductive glass core surrounded by an externally reflecting PBG structure containing a resonant cavity. (b) An integrated self-heat-monitoring hollow-core PBG fiber transmits a high-power laser beam while monitoring temperature inside the fiber by MSM thin-film heat sensors. (c) A dual electron-photon fiber transmits light through a hollow core surrounded by a mirror structure and electrical signal via metallic microwires along the length of the fiber. (d) Nonlinear, all-solid, nanostructured chalcogenide glass/polymer fibers for supercontinuum light generation.

core and a two-dimensional (2-D) photonic crystal cladding, generates supercontinuum light at desired wavelengths when the highly nonlinear core is pumped with a laser [11].

\section{Preform-to-Fiber Fabrication APPROACH}

The process of optical-fiber drawing from a solid structured preform that is heated and deformed in the viscous state has been well established through the development of the fiber telecommunications industry and enables the rapid fabrication of kilometer-scale continuous lengths of fiber with precise dimensional tolerances. The emerging field of microstructured silica [12]-[14] and polymer [15], [16] optical fibers, comprised of a single material combined with air holes, relies on the controlled fabrication of submicrometer features.

However, our strategy is to produce multimaterial composite fibers and fiber-based devices having optical, electrical, and thermal functionalities at nanometer feature sizes and kilometerlength scales. This goal necessitates innovative fabrication approaches that allows the use of new materials and geometries that have not been, hitherto, associated with fibers. By combining materials and exploring novel geometries, conventional functionalities can be enhanced and new ones introduced. This vision presents a unique set of challenges. Irrespective of the processing method, materials with widely disparate electronic and optical properties must be compatible in such a way that enables the production of kilometers of fiber with submicrometer feature sizes, having an aspect ratio of the order of $10^{10}$.

Our core strategy is to use the familiar technique of thermal drawing a macroscopic fiber preform in the viscous state. This technique guarantees the production of kilometers of fibers at low cost and high uniformity. Several constraints, however, are imposed by the very nature of this technique, specifically in the choice of materials that are amenable to this type of processing. In particular, we discuss the use of a materials system that combines chalcogenide glasses, organic polymers, and metallic alloys in a macroscopic preform rod.

\section{A. Material Selection}

The main requirements in the materials used in these fibers are summarized as follows [17].

1) In general, materials should be glassy in order to be drawn at reasonable speeds in the fiber-drawing process with self-maintaining structural regularity. Thus, our materials (or at the very least, the majority component) must not simply be amorphous when deposited or made into a preform, but must remain amorphous and not crystallize when cycled through softening and drawing temperatures.

2) The materials must be above their respective softening points at overlapping temperatures to enable fiber codrawing.

3) The materials should exhibit compatible viscosities at the processing temperatures of interest and should exhibit good adhesion/wetting in the viscous and solid states without cracking even when subjected to thermal quenching.

4) For the photonic bandgap fibers, the selected materials should exhibit low optical absorption over a common wavelength band. The penetration depth through multilayer structure must be smaller than the absorption length.

5) The metals should be molten at the drawing temperature during the fiber-drawing process. Even though molten metals have very small viscosities, the capillary drawing confines the molten metals between high viscosity semiconducting and insulating interfaces.

Various high-refractive-index chalcogenide glasses, lowrefractive-index high-temperature thermoplastics, and lowmelting-point metals and alloys are identified as potential candidates based on their optical and electrical properties, overlapping thermal softening regimes, and their amenability to film processing. Chalcogenides [18], [19] are high-index inorganic glasses that contain one or more of the chalcogen elements. These glasses are typically made of arsenic, sulfur, selenium, tellurium, germanium, tin, and gallium elements. Chalcogenide glasses have been explored due to their unique optical properties such as high nonlinearity [20], wide transparency window at near- and mid-infrared wavelengths (up to $25 \mu \mathrm{m}$ or higher), and high refractive indices (between 2.2 and 3.6). In particular, As-based chalcogenide glasses have low softening temperatures (between $100{ }^{\circ} \mathrm{C}$ and $300^{\circ} \mathrm{C}$ ), high refractive index, and infrared transparency. Due to these unique properties, these glasses become an important class of materials in multilayer PBG fiber manufacture [3], [4], [7].

In selecting a low-refractive-index $(n=1.3-1.7)$ component as the second material for these PBG fibers, it is of principal concern to match the thermal properties of some of the chalcogenide glasses. Thermoplastic polymers tend to have lower softening temperatures than typical chalcogenide glasses; however, a few candidates have been identified with thermal properties that are comparable to chalcogenides. Disadvantages of polymeric materials that may need to be addressed include 
TABLE I

THERMAL AND OPTICAL PROPERTIES OF GLASSES AND POLYMERS

\begin{tabular}{lllll}
\hline \multicolumn{1}{c}{ Materials } & $T_{g}\left({ }^{\circ} \mathrm{C}\right)$ & $\begin{array}{c}n(\text { at } 1.5 \mu \mathrm{m}) \\
n_{0}-\mathrm{i} k\end{array}$ & $\begin{array}{c}n(\text { at } 10.6 \mu \mathrm{m}) \\
n_{0}-\mathrm{i} k\end{array}$ & $\begin{array}{l}\text { Transparency } \\
\text { Window }(\mu \mathrm{m})\end{array}$ \\
\hline $\mathrm{As}_{2} \mathrm{~S}_{3}$ & 190 & 2.40 & 2.34 & $0.6-11$ \\
$\mathrm{As}_{2} \mathrm{Se}_{3}$ & 185 & 2.77 & 2.71 & $0.8-15$ \\
$\mathrm{Ge}_{15} \mathrm{As}_{25} \mathrm{Se}_{15} \mathrm{Te}_{45}$ & 190 & 3.32 & & $1.5-16$ \\
$\mathrm{PES}$ & 220 & $1.62-\mathrm{i} 0.0001$ & $1.65-\mathrm{i} 0.0047$ & $0.3-2.1^{\mathrm{a}, \mathrm{b}}$ \\
$\mathrm{PEI}$ & 215 & $1.65-\mathrm{i} 0.0017$ & $1.66-\mathrm{i} 0.048$ & $0.3-2.1^{\mathrm{a}, \mathrm{b}}$ \\
$\mathrm{PS}$ & 190 & 1.63 & & $0.3-2.1^{\mathrm{a}, \mathrm{b}}$ \\
\hline
\end{tabular}

The refractive indices were measured by using a Sopra GES5 UV-Vis-IR spectroscopic ellipsometer [21].

${ }^{a}$ The transmittance is $90 \%$ or higher for $50-\mu \mathrm{m}$-thick film.

${ }^{\mathrm{b}}$ The $k$ values are relatively low (transmittance is $65 \%$ or higher for $50-\mu \mathrm{m}$-thick film) between 2.1-6.0, 9.3-11.3, and 12.2-13.6 $\mu \mathrm{m}$.

their thermal stability and optical absorption. Nevertheless, the wide variety of polymers available, the feasibility of processing them in film form, and their excellent mechanical toughness make these materials our principal candidates for combination with chalcogenide glasses in composite PBG fibers [17]. In general, we used three different polymers, namely polyethersulfone (PES), polyetherimide (PEI), and polysulfone (PS). These polymers have similar coefficient thermal expansion (CTE) compared to that of $\mathrm{As}_{2} \mathrm{Se}_{3}$ and $\mathrm{As}_{2} \mathrm{~S}_{3}$ glasses. Table I shows the thermal and optical properties of some of the chalcogenide glasses and polymers.

Many elemental metals, alloys, as well as eutectics, can be incorporated with the above polymers and chalcogenide glasses; for instance, $\mathrm{Sn}\left(T_{\mathrm{M}}=232^{\circ} \mathrm{C}\right), \mathrm{Bi}\left(T_{\mathrm{M}}=271^{\circ} \mathrm{C}\right)$, In $\left(T_{\mathrm{M}}=\right.$ $\left.157^{\circ} \mathrm{C}\right)$, Sn $(96 \%)-\mathrm{Ag}(4 \%)\left(T_{\mathrm{M}}=221^{\circ} \mathrm{C}-229^{\circ} \mathrm{C}\right)$, and $\mathrm{Pb}$ (38\%)-Sn (62\%) $\left(T_{\mathrm{M}}=183^{\circ} \mathrm{C}\right)$. Depending on the drawing temperature, we choose a suitable candidate from the above.

\section{B. Material Synthesis}

The amorphous semiconductor glasses As-Se [22], As-SeTe-Sn [5], and Ge-As-Se-Te [23]-[25] are prepared from highpurity $\mathrm{Ge}, \mathrm{As}, \mathrm{Se}, \mathrm{Te}$, and $\mathrm{Sn}$ elements using conventional sealed-ampoule melt-quenching techniques. The materials were weighed and placed into a quartz tube under a nitrogen atmosphere. The tube was heated to $330^{\circ} \mathrm{C}$ for an hour at a rate of $1{ }^{\circ} \mathrm{C} /$ min under vacuum to remove surface oxides, and cooled to room temperature at the same rate. The ampoule was formed by sealing the tube under vacuum $\left(10^{-5}\right.$ torr). Depending on the actual composition, it was then heated to $800^{\circ} \mathrm{C}$ [ for $\mathrm{As}_{2} \mathrm{Se}_{3}$ and $\mathrm{As}_{40} \mathrm{Se}_{50} \mathrm{Te}_{10} \mathrm{Sn}_{5}$ (AST-Sn)] or $900^{\circ} \mathrm{C}$ [for $\mathrm{Ge}_{15} \mathrm{As}_{25} \mathrm{Se}_{15} \mathrm{Te}_{45}$ (GAST)] at a rate of $2{ }^{\circ} \mathrm{C} / \mathrm{min}$ in a rocking furnace, while held vertical for $24 \mathrm{~h}$, and then rocked for $12 \mathrm{~h}$ to increase mixing and homogenization. The glass liquid was cooled to $600^{\circ} \mathrm{C}\left(700^{\circ} \mathrm{C}\right.$ for GAST) in the furnace, and then quenched in (cold for GAST) water. Subsequently, it was annealed for half an hour near the glass transition temperature before being cooled gradually to room temperature. The $\mathrm{As}_{2} \mathrm{Se}_{3}$ glass used in supercontinuum generation was further purified by distillation. Using this procedure, mechanically stable glass rods with diameters ranging 5-18 $\mathrm{mm}$ and lengths $10-18 \mathrm{~cm}$ were obtained.

Commercially available polymer films are purchased from several suppliers: 25-, 50-, 75-, and 125- $\mu \mathrm{m}$-thick PES from Westlake Inc., Lititz, PA; 8-, 10-, 13-, and 25- $\mu$ m-thick PEI

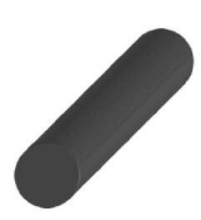

(a)

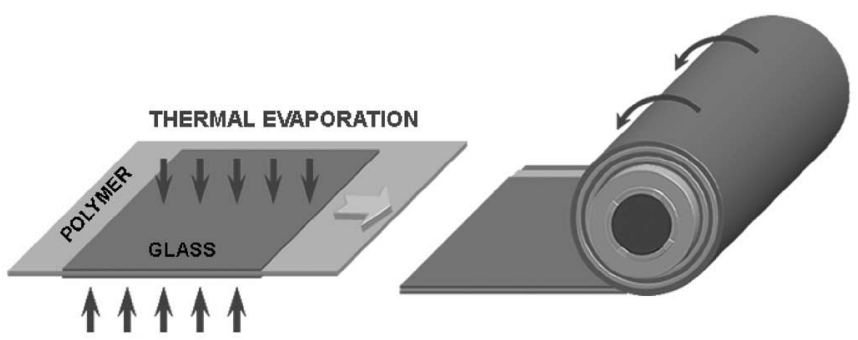

(d)

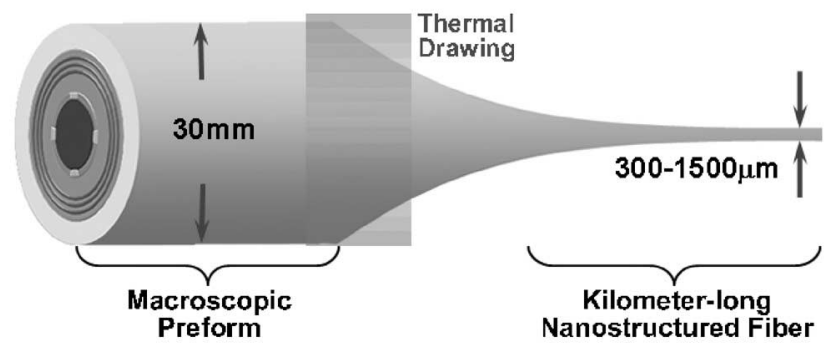

(f)

Fig. 2. Fabrication steps for nanostructured fibers and fiber devices. (a) An amorphous glass is synthesized from elements in an evacuated quartz tube. (b) The glass rod is assembled with an insulating polymer shell and four metal electrodes. (c) A polymer sheet is rolled around the structure to confine the metal conduits inside the polymer. (d) A high-refractive-index glass is thermally evaporated on both sides of a meter-long thin polymer film uniformly. (e) The evaporated film is rolled around the structure obtained in (c). The final structure is then thermally consolidated in a vacuum oven in order to get a solid preform rod. (f) The preform is thermally drawn to kilometer-long mesoscopic-scale fibers containing micro- and nanostructures.

from Nagai, Japan; and 25-, 50-, and 75- $\mu$ m-thick PS from Ajedium, Newark, DE.

\section{Fabrication Procedure}

We now discuss the fabrication of a structured preform essentially consisting of a semiconductor core (a chalcogenide glass), metallic electrodes (tin), and multiple alternating thin films (omnidirectional PBG structure) that are uniform and in intimate contact with each other. Even though the fabrication procedure varies for the different structures proposed in Fig. 1, we describe the overall approach in our discussion of the spectrally tunable fiber photodetecting device [Fig. 1(a)]. A schematic outline of the steps involved in fabricating the preform is shown in Fig. 2, and are as follows.

1) A semiconductor rod is synthesized from elemental batch components in an evacuated quartz ampoule at $700^{\circ} \mathrm{C}-900^{\circ} \mathrm{C}$ in a rocking furnace [22] [Fig. 2(a)].

2) A polymer tube, obtained from a thin film by rolling on a Teflon rod, is consolidated in a vacuum oven. Four slits are opened, and the metallic electrodes are placed in these openings [Fig. 2(b)]. Several layers of polymer film were rolle in order to confine the metallic electrodes [Fig. 2(c)]. 


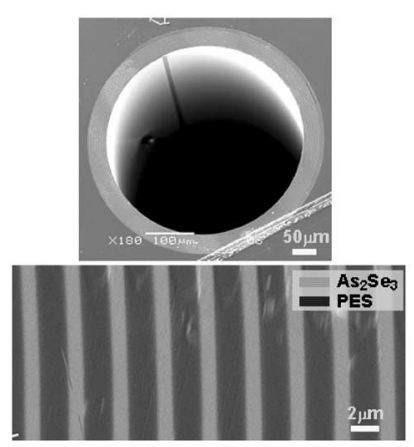

(a)

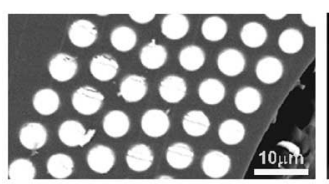

(c)

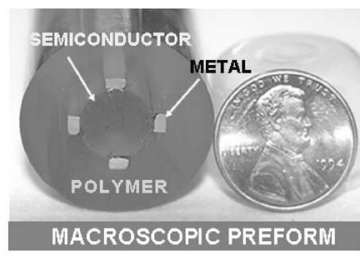

(f)

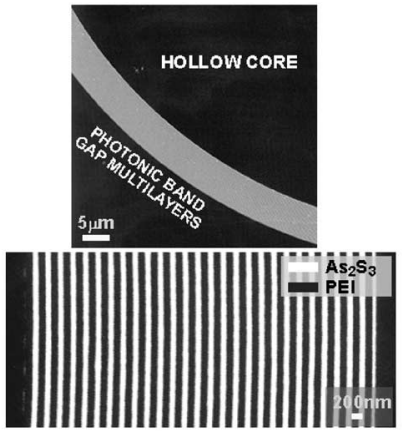

(b)

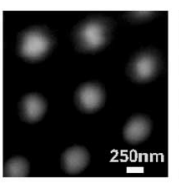

(d)

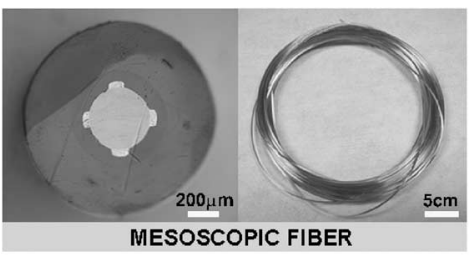

(g)

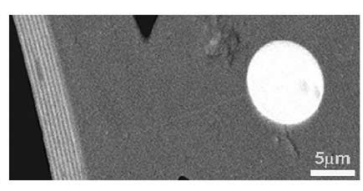

(e)
Fig. 3. Examples of fibers and fiber devices produced by using the preform-tofiber technique. Hollow-core, all-dielectric omnidirectional PBG fibers to guide laser light at different wavelengths. (a) $10.6 \mu \mathrm{m}$. (b) $1.06 \mu \mathrm{m}$. (c) Hexagonal photonic crystal structure in fiber. A high-refractive-index glass (bright region) is embedded in a low-index polymer matrix. (d) Kilometer-long amorphous semiconducting nanowires in a polymeric fiber. (e) Integration of optical (PBG multilayer mirror, left) and electrical (Sn microwire, right) elements in a fiber. (f)-(h) Fiber-based MSM light or heat sensors. Successful thermal drawing of a multimaterial preform yields the following. (g) Functional mesoscopic devices. (h) Functional mesoscopic devices in large length scale.

3) The thin-film deposition technique that we settled on was a thermal evaporation of the chalcogenide glass onto freestanding, commercially obtained polymer films, allowing us to deposit layers of the chalcogenide glass at high deposition rates [Fig. 2(d)]. We deposited uniformly 1.5$15-\mu \mathrm{m}$-thick chalcogenide glasses on both sides of meterslong, 20-cm-wide, and 8-50- $\mu \mathrm{m}$-thick polymer films.

4) After rolling several polymer cladding layers, the preform was consolidated in a zone-defined horizontal vacuum oven while rotating the preform [Fig. 2(e)].

5) The preform was then thermally drawn into hundreds of meters of fibers in a three-zone vertical tube furnace [Fig. 2(f)]. The position of the PBG was measured by a Fourier transform infrared (FTIR) spectrometer (Bruker, Tensor 37) during the drawing process.

\section{Examples}

One class of fibers produced using the above-mentioned technique is wavelength scalable hollow-core omnidirectional PBG fibers. Fig. 3(a) shows the scanning electron microscope (SEM) micrograph of a fiber having 11 cylindrical bilayers of alternating $\mathrm{As}_{2} \mathrm{Se}_{3}$ and PES with thicknesses of 1.07 and $1.96 \mu \mathrm{m}$, respectively. This fiber has a fundamental bandgap centered at 10.6- $\mu \mathrm{m}$ wavelength, which was designed to transport a highpower $\mathrm{CO}_{2}$ laser beam. At the preform level, a 13- $\mu$ m-thick $\mathrm{As}_{2} \mathrm{Se}_{3}$ film was uniformly deposited on both sides of a $45-\mu \mathrm{m}$ thick, 22-cm-wide, and 1-m-long PES polymer film, and then rolled onto a teflon tube. The initial geometry and layer thickness ratio can be preserved during the fiber-drawing process if suitable drawing conditions are chosen. Since the position of the bandgap of a multilayer mirror scales with layer thickness, one can obtain PBG guidance at shorter wavelengths by reducing the fiber's outer diameter. As shown in Fig. 3(b), the fiber can transport Nd-YAG laser light centered around $1.06 \mu \mathrm{m}$ with $\mathrm{As}_{2} \mathrm{Se}_{3}$ and PEI layer thicknesses of 101 and $160 \mathrm{~nm}$, respectively.

The second class of fibers contains 2-D photonic crystal structures [Fig. 3(c) and (d)]. The preform of these fibers was prepared by multiple thermal drawing as follows.

1) A chalcogenide glass $\left(\mathrm{As}_{2} \mathrm{Se}_{3}\right)$ rod was synthesized.

2) The first preform with $\mathrm{As}_{2} \mathrm{Se}_{3}$ core and PES cladding was constructed, and then subsequently drawn into $500-1000-\mu$ m-thick fibers.

3) $800-\mu \mathrm{m}$-thick fibers were cut into 20 -cm pieces, and then these fibers were used to construct the second preform having a hexagonal photonic crystal structure.

4) The final perform was drawn into $100-900-\mu$ m-thick fibers in a three zone tube furnace at $295^{\circ} \mathrm{C}$ (middle zone). At the end of two consecutive drawings, it was possible to reach a reduction factor (initial preform feature size/final fiber feature size) of the order of $5 \times 10^{5}$. The SEM micrograph of kilometerlong semiconducting nanowires with diameter around $200 \mathrm{~nm}$ in a polymer matrix, is shown in Fig. 3(d). We expect that one can reach an ultimate length scale less than $20 \mathrm{~nm}$ in such a material system [17].

The third class of structures integrates optical and electrical elements in the same fiber. As shown in Fig. 3(e), a cylindrical multilayer optical mirror was combined with a micrometer-scale wire, which ran along the fiber length. The fourth class integrates fiber-based functional devices containing metallic, semiconducting, and insulating elements in a variety of geometries. Fig. 3(f) shows a macroscopic preform rod containing MSM devices that sense light or heat depending on the semiconductor core. The fiber device [Fig. 3(g)] is connected to external circuitry through metallic electrodes running along meters-long fiber [Fig. 3(f)]. Optical or thermal excitation on any point along the fiber device changes the electrical conductivity.

\section{MSM FIBER PHOTODETECTORS}

Light detection on curved surfaces and large areas is a task that has yet to be achieved in a convenient way. We present a new methodology for light detection on large areas by using flexible, low-cost, lightweight, mechanically tough, and arbitrarily long one-dimensional (1-D) photodetectors [5], [6], [10]. Underlying this approach is a new fiber structure that senses impinging light along its entire length and generates an electrical signal.

The photodetecting fibers were obtained from a macroscopic preform (33-mm-thick and 20-cm-long) consisting of a cylindrical semiconductor chalcogenide glass core (AST-Sn), contacted 

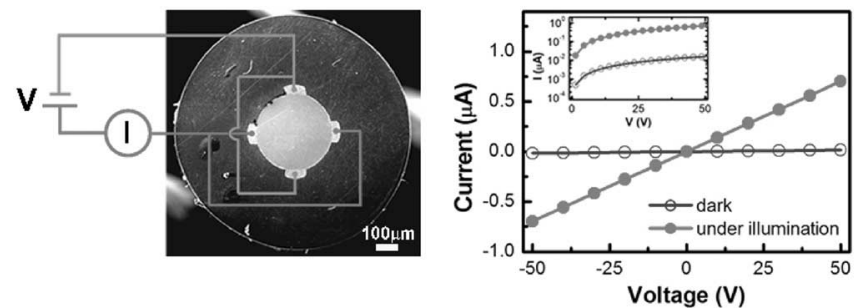

(a)

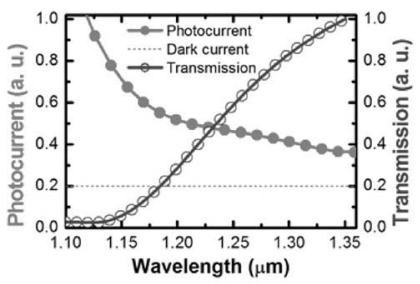

(c)

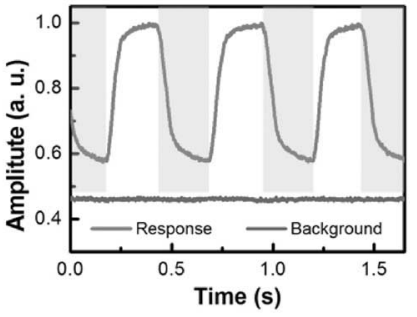

(e)

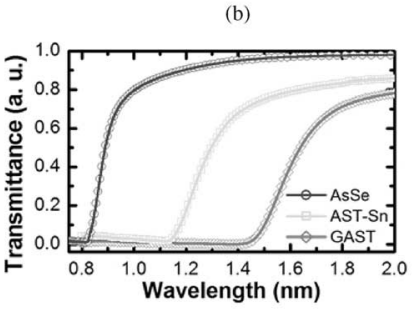

(d)

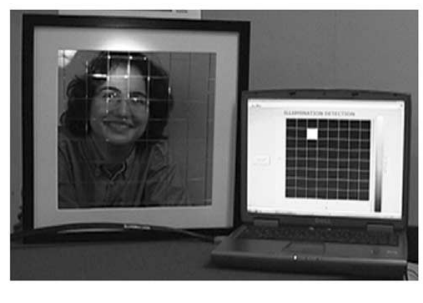

(f)

Fig. 4. MSM fiber photodetectors. (a) SEM micrograph of the entire cross section of an MSM device. The device is connected to the external circuitry through four metallic electrodes running along the fiber length. (b) The $I-V$ characteristics of the MSM fiber photodetector in the dark and under illumination. (c) Measured photocurrent sweeping wavelength of tunable OPO while keeping the optical power constant, and transmission spectrum of bulk Sn-doped AST chalcogenide glass. (d) Tunability of the operation wavelength by changing the glass composition. (e) Temporal response of the fiber device. (f) A 2-D fiber web can be used to track a point of illumination in real time.

by four Sn metal conduits that are encapsulated in a protective PES cladding. This highly photoconductive AST-Sn glass is optimized subject to the following requirements: 1) large photoconductivity in the spectral range of interest; 2) the viscosity range should be compatible with the polymer; and 3) an enhanced stability against crystallization during fiber drawing. The preform was consolidated in a three-zone horizontal tube furnace at $260^{\circ} \mathrm{C}$ under vacuum $\left(10^{-3}\right.$ torr $)$ while rotating the preform along its axis, and was subsequently heated and drawn into hundreds of meters of fiber. The preform geometry was preserved throughout all thermal processes, i.e., consolidation and drawing. The fiber exhibits both electrical and optical functionalities that follow from the excellent contact and appropriate element dimensions. Fig. 4(a) shows the cross section of a 980- $\mu$ m-thick fiber, which is connected to an external circuit though the metallic electrodes. The electrical conductance of this fiber was found to increase dramatically upon illumination by white light [by two orders of magnitude for $20-\mathrm{mW}$ incident power, see Fig. 4(b)]. The current-voltage $(I-V)$ curve reveals clear ohmic response both in the dark and under illumination.

Next, we characterized the broadband photoconductive response of the device fiber [Fig. 4(c)]. The fiber was illuminated externally with a laser beam from a tunable, synchronously

pumped optical parametric oscillator (Mira OPO, Coherent). The optical transmission of a bulk AST-Sn glass sample (12-mm-diameter and 5-mm-length) was obtained via an FTIR measurement [Fig. 4(c)]. A 50-V dc voltage was applied to the fiber and the current was measured using a picoampere meter (Yokogawa/Hewlett Packard 4140B). The spectral photoconductive response is commensurate with the optical transmission measurement. Note that changing the chemical composition of the glass core can change the spectral characteristics of the photoconductive response, as seen in Fig. 4(d), where the optical transmission spectra corresponding to $\mathrm{As}_{2} \mathrm{Se}_{3}$, AST-Sn, and GAST are plotted. The onset of optical transmission, and hence the drop in the photoconductivity, is shifted by changing the glass composition. Indeed, the photoconductive glass in the core may be substituted with other families of glasses that are sensitive to other physical quantities, such as temperature [9] or chemical contaminants. An example of the temporal response of the MSM device fiber is depicted in Fig. 4(e).

While the individual fiber behaves as a 1-D photodetector with sensitivity to visible and infrared light at every point along its entire length, it is the assembly of such fibers into 2-D grids or photodetector fiber webs that enables the detection of an illumination point. Moreover, this grid achieves $N^{2}$ detection resolution with only $2 N$ elements. An example of such a fiber web used to measure the coordinates of an illumination point on a $30 \times 30 \mathrm{~cm}$ area consisting of 64 resolved points is shown in Fig. 4(f). The web is made of 16 fibers arranged in an $8 \times 8$ grid. When light is incident on any of the fibers, a photocurrent is generated, and the voltage on the fiber changes. Each fiber is connected to an operational amplifier (in unity-gain configuration) that matches its impedance to that of a data acquisition card placed in a PC. A computer program monitors the voltages supplied (16 in total), which indicate the amount of light incident on each fiber. The program first identifies the voltages related to the horizontal and vertical fibers, and then identifies the location of the light beam. When no light is incident on the grid, the voltage drops on the fibers assume their "dark" values, and the "virtual grid" on the computer screen is uniformly black. The horizontal (vertical) fiber that undergoes the maximum change in voltage determines the vertical (horizontal) coordinate of the incident beam. A white spot is then placed on the virtual grid at the location of the horizontal and vertical coordinates thus determined. The voltage on the fibers is monitored continuously, and the refresh rate is limited by the relaxation time of the fibers and the speed of the data acquisition card.

\section{SPectrometric Photodetecting Fibers}

One may integrate a PBG resonant structure with a photodetecting fiber to produce a narrow-band tunable fiber photodetector [Fig. 1(a)]. The PBG structure contains an optical cavity and is introduced in the optical path shielding the photoconducting core from ambient illumination sources. Upon external illumination, when the wavelength of the radiation matches that of the cavity resonance, one observes an electric response from the fiber core thus establishing the spectroscopic functionality of the fiber [5]. 


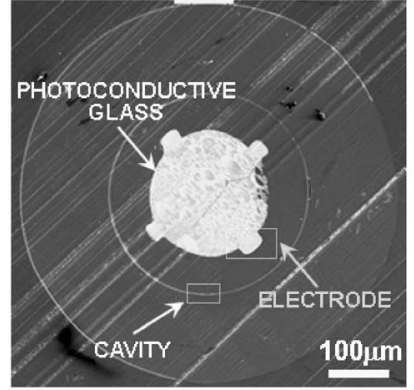

(a)

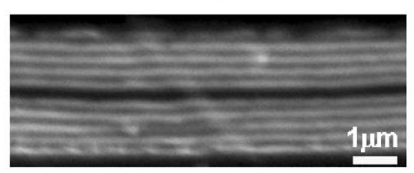

(b)

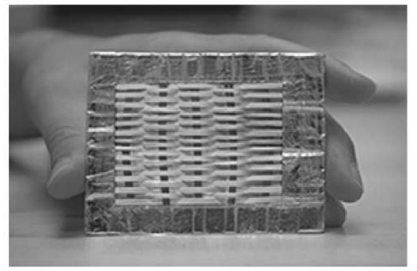

(c)

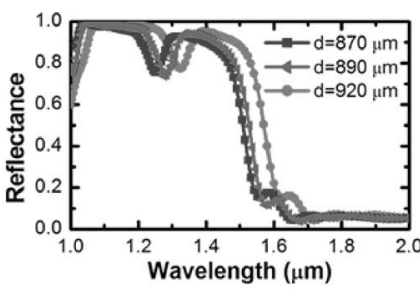

(d)

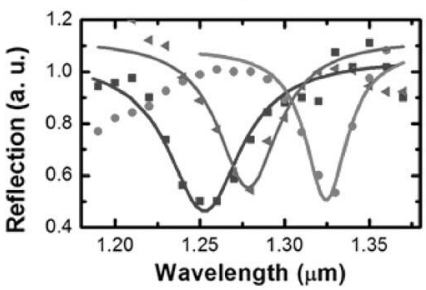

(e)

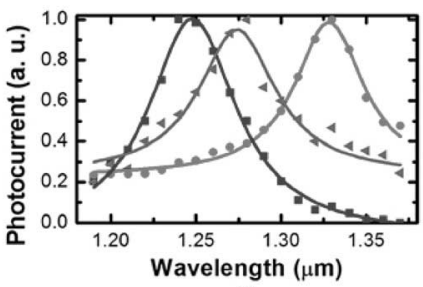

(f)

Fig. 5. Integrated optoelectronic device fiber. (a) SEM micrograph of the entire cross section of a $650-\mu \mathrm{m}$-thick device fiber with $200-\mu \mathrm{m}$ chalcogenide glass core surrounded by a PES cladding. The core region is surrounded by a resonant cavity structure. (b) A magnified micrograph showing the cavity structure. (c) Spectrometric fiber mesh-grid. (d) Broadband FTIR spectra of device fibers with different outer diameters. (e) Measured back-reflected light power from the same three device fibers when illuminated with a tunable OPO laser beam. (f) Simultaneously measured photocurrents through the device fibers.

The preform of this spectroscopic fiber consists of a photoconductive chalcogenide glass rod (AST-Sn), having diameter $12 \mathrm{~mm}$ and length $18 \mathrm{~cm}$, contacted by four $\mathrm{Sn}$ conduits, and surrounded by a quarter-wave $\mathrm{As}_{2} \mathrm{Se}_{3} / \mathrm{PEI}$ multilayer mirror structure with a $\lambda / 2$ PEI cavity and a protective PES cladding. The preform was consolidated in a three-zone horizontal tube furnace at $260^{\circ} \mathrm{C}$ under vacuum $\left(10^{-3}\right.$ torr $)$ while rotating the preform along its axis. Subsequently, the preform was drawn in a three-zone vertical tube furnace at a top zone temperature between $185^{\circ} \mathrm{C}$ and $230^{\circ} \mathrm{C}$ and a middle zone temperature $295^{\circ} \mathrm{C}$. A capstan speed of about $0.7-3 \mathrm{~m} / \mathrm{min}$ produced a fiber of a diameter between 1200 and $500 \mu \mathrm{m}$, and a length of several hundred meters. Fig. 5(a) shows SEM micrographs of the spectroscopic fiber cross section. The resonant PBG structure encapsulating the MSM photodetecting devices is shown in Fig. 5(b). For a 920- $\mu$ m-thick fiber, the thicknesses of $\mathrm{As}_{2} \mathrm{Se}_{3}$ and PEI layers are 117 and $204 \mathrm{~nm}$, respectively. The photograph in Fig. 5(c) is a spectrometric mesh-grid woven from fibers having different outer diameters; each strand in the mesh-grid can detect a specific wavelength that matches the resonance frequency of its Fabry-Perot structure.

The resonance wavelength and the PBG are determined by the fiber outer diameter. From the fabricated fibers of various diameters, we selected three fibers of outer diameters 870,890 , and $920 \mu \mathrm{m}$. The reflectivity of the optical cavity structures were

measured with an FTIR spectrometer (Nicolet/SpectraTech, NicPlan infrared microscope and FTIR [Magna 860]) and are displayed in Fig. 5(d). The FTIR spectra agree well with the calculated spectra when averaging over the range of angles admitted by the microscope objective $\left(0^{\circ}\right.$ to $\left.35^{\circ}\right)$ is taken into consideration, leading to a reduction in the apparent quality factor of the cavity mode [26]. The primary bandgap of the mirror structure extends from 1 to $1.6 \mu \mathrm{m}$.

In order to characterize the optoelectronic response of our integrated device fiber, it is useful to measure both the electrical photocurrent and the optical reflectivity simultaneously. This can be achieved by externally illuminating the three aforementioned fibers with a laser and measuring the back-reflected light though a beam splitter, while simultaneously monitoring the generated photocurrent in the photosensitive core. For optical characterization, we used a Verdi10 (Coherent) to pump a Ti-S femtosecond laser (Mira 900, Coherent) that was then down-converted using a synchronously pumped optical parametric oscillator (OPO) (Mira OPO, Coherent). The OPO beam was focused onto the outer surface of the fiber using a $\times 5$ microscope objective (NA $=0.1$ ), and the back-reflected light from the fiber was directed through a beam splitter to an InGaAs photodetector (Newport 818-IG). The optical power incident on the fiber surface was maintained at $30 \mathrm{~mW}$ using a variable optical attenuator, while the wavelength of the laser beam was swept. For simultaneous electrical characterization, we measured the current flowing through the fiber electrodes using a pico-ampere meter (Yokogawa/Hewlett Packard 4140B). The dc voltage difference applied to the two fiber electrodes was $50 \mathrm{~V}$. At each wavelength, the incident optical power was adjusted, the electrical current was recorded, and the back-reflected light power measured.

Fig. 5(e) shows the measurement results of the back-reflected light from these fibers as the wavelength of the laser (the tunable OPO) is swept. Concurrently, we plotted the results of photocurrent measurements of these fibers [Fig. 5(f)]. At the resonance wavelength, the back-reflection is diminished, and the light reaches the photoconductive core. Consequently, the corresponding photocurrent is enhanced. This integrated optoelectronic fiber device functions as a narrowband photodetector, with the detected wavelength tuned through the fiber outer diameter.

\section{Self-Heat-Monitoring InTEGRATEd Fibers For High-POWER LASER BEAM DELIVERY}

The ability to integrate optical transport and thermal monitoring for failure prediction is of paramount importance if high-power optical transmission lines [27]-[29] are to be operated safely and reliably in medical, industrial, and defense applications [30]. Recently, the use of hollow-core PBG fibers for in vivo endoscopic surgery on human patients and canine larynges has been successfully demonstrated [31]. However, fibers used for laser surgery transport significant laser power through their core and even a small defect nucleating within such a high-power optical transmission line can result in unintentional energy release with potentially detrimental or catastrophic consequences. 

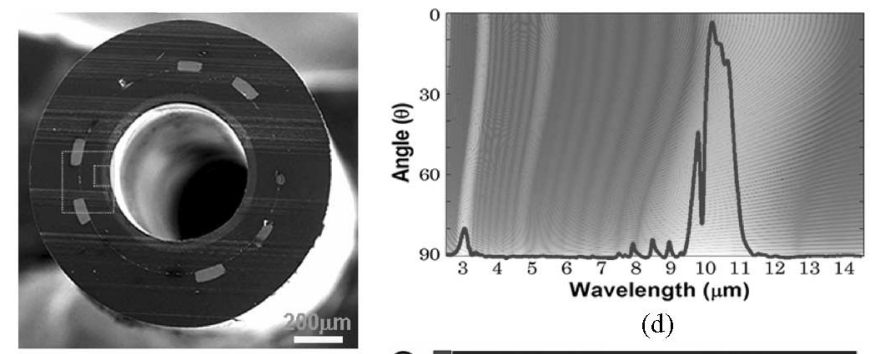

(d)

(a)
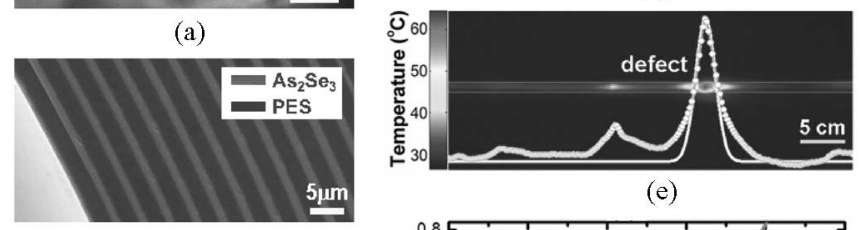

(e)

(b)

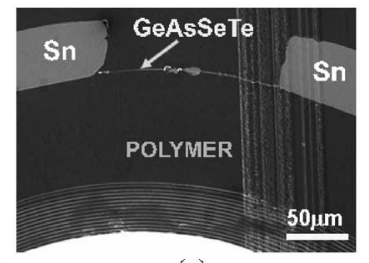

(c)

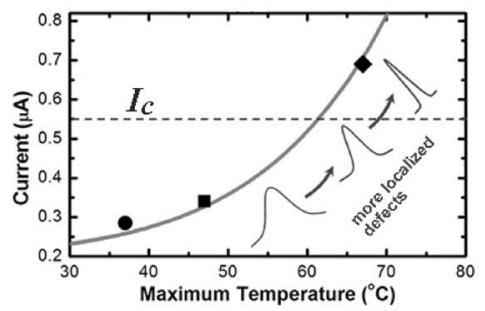

(f)

Fig. 6. SEM micrographs of the integrated self-heat-monitoring fiber. (a) Entire cross section. (b) Cylindrical omnidirectional Bragg mirror. (c) MSM heat sensor. (d) Calculated band diagram of the cylindrical multilayer PBG structure and the corresponding measured broadband transmission spectrum (solid line). (e) Thermal photograph of a fiber containing a single localized defect, along with the measured (circles) and fitted (solid line) temperature distribution along the fiber. (f) Calculated current as a function of the maximum temperature along the fiber for a constant dissipated power (solid line) and three experimental points.

We designed and fabricated a fiber device structure [Fig. 6(a)], which contains integrated optical, electrical and thermal elements for self-monitored optical transport [8]. This multimaterial fiber comprised of a polymer insulator (PES), a binary large bandgap semiconductor $\left(\mathrm{As}_{2} \mathrm{Se}_{3}\right)$, a quaternary narrow bandgap semiconductor (GAST), and metallic elements $(\mathrm{Sn})$ is drawn at high speeds from a single fiber preform to produce extended lengths of optically and thermally functional fibers. An optical transmission element, which is a hollow-core multilayer [Fig. 6(b)] cylindrical PBG structure [32], is designed to guide high-power radiation at $10.6 \mu \mathrm{m}$ along the fiber axis [4]. Multiple thermal-detection elements [Fig. 6(c)], are placed in the vicinity of the hollow core for the purpose of temperature monitoring along the entire fiber length. Metal wires bridged by a temperature-sensitive semiconductor layer extend along the length of the fiber and deliver an electrical response to the fiber ends upon change in the fiber temperature.

The macroscopic preform rod, which shares the final fiber geometry, was prepared by the following steps.

1) A 13- $\mu$ m-thick film of $\mathrm{As}_{2} \mathrm{Se}_{3}$ was thermally evaporated on both sides of a 50- $\mu \mathrm{m}$-thick, 24-cm-wide, and 40-cmlong PES film that was then rolled onto a 14.2-mm-thick teflon FEP rod, which does not stick to the inner glass layer during thermal processing steps.

2) The $10-\mu \mathrm{m}$-thick GAST film was then deposited by thermal evaporation with a vacuum evaporator (Ladd Indus-

tries) on one side of a 50- $\mu \mathrm{m}$-thick PES film. After rolling a few-millimeters-thick buffer PES layer, the single GAST layer was rolled.

3) This temperature-sensitive GAST layer was contacted by six Sn (tin) metal conduits (0.8-mm-thick, 2.5-mm-wide, and $15-\mathrm{cm}-\mathrm{long}$ ) that are encapsulated in a protective PES cladding.

4) The preform was consolidated for $70 \mathrm{~min}$ at $260^{\circ} \mathrm{C}$ under vacuum $\left(10^{-3}\right.$ torr) in a three-zone horizontal tube furnace, and the teflon rod was removed from the core after consolidation. The preform was annealed and then cooled gradually to room temperature.

The preform was subsequently heated and drawn into tens of meters of fiber in a draw tower (Heathway). Fibers were drawn at the central zone of a three-zone vertical tube furnace (Thermcraft) with the top-zone temperature of $190^{\circ} \mathrm{C}$ and the middle-zone temperature of $295^{\circ} \mathrm{C}$. The fiber diameter was monitored with laser diameter monitors and the target fiber diameter was determined by measuring broadband FTIR spectra during drawing.

We first discuss the optical transport properties of this hybrid fiber. The fiber has a $1270-\mu \mathrm{m}$-thick outer diameter and a $560-\mu \mathrm{m}$ diameter hollow core surrounded by a multilayer structure consisting of 13 bilayers of alternating $\mathrm{As}_{2} \mathrm{Se}_{3}$ and PES having thicknesses of 1 and $1.9 \mu \mathrm{m}$, respectively [see Fig. 6(b)]. The calculated PBG diagram of the hybrid fiber is depicted in Fig. 6(d), revealing an omnidirectional bandgap extending from 9.4 to $11.4 \mu \mathrm{m}$. The light gray areas represent guided modes inside the core, while the dark gray areas correspond to regions where light is not guided, but instead radiates through the multilayer structure. The transmission spectrum of a 1-m-long fiber was measured by the FTIR spectrometer and is overlaid on the band diagram in Fig. 6(d).

To demonstrate the delivery of high-power laser light through the hybrid fiber while monitoring the temperature in the fiber, a $\mathrm{CO}_{2}$ laser (GEM-25, Coherent-DEOS) at $10.6 \mu \mathrm{m}$ was coupled to the fiber and a $50-\mathrm{V}$ dc voltage was applied to the device electrodes. The input and output optical power as well as the current through the electrodes were recorded. We measured the power radiated from the fiber outer surface and also obtained thermal images using an infrared (IR) camera (FLIR). The power escaping from the surface of the fiber was found to be negligible with respect to the overall power loss, suggesting that the difference in power between the input and output dissipates in the fiber cladding and converts into heat.

In order to investigate the self-fault-detection capabilities of our fiber, we measured the current as a function of the dissipated power for three 40-cm-long fibers: 1) a defect-free; 2) a bent defect-free; and 3) a single-defect-containing fiber. The defect on the fiber was intentionally generated by burning a small spot on the fiber with a $\mathrm{CO}_{2}$ laser beam. The temperature distribution along the single-defect-containing fiber is shown in Fig. 6(e). While indeed power is dissipated throughout the entire fiber length, it is the localized defect site that undergoes the most significant heating [Fig. 6(e)]. This heating, in turn, causes an exponential increase in the semiconductor's conductivity, which results in an increase in the current flow [Fig. 6(f)]. The three 
experimental points shown in Fig. 6(f) correspond to a defectfree straight fiber (circle), a bent fiber (square), and a singledefect-containing straight fiber (diamond). The current exceeds a threshold value $I_{\mathrm{c}}$ only for the defect case. Thus, the exponential dependence on temperature of the electrical conductivity of the semiconducting material allows for the discriminationin real time-between the normal transmission conditions and those that are indicative of localized defect formation, enabling for the first time a self-monitoring high-power optical transmission line for failure prediction and prevention.

Incorporating a thermal element [8], [9] with optical structures may potentially yield various optoelectronic devices such as fiber-based tunable mid-infrared attenuators and thermo-optic switches [33]. We expect that flexible, thin, low-cost, large-area, distributed, mechanically tough, and polymeric fiber-based heat sensors might be used in medical, military, industrial, and space applications.

\section{HyBRID ELECTRON-PHOTON TRANSPORT FIBERS}

Conventional optical fibers guide light in a solid core via total internal reflection. This process has fundamental limitations stemming from light absorption by electrons and phonons, material dispersion, Rayleigh scattering, thermal lensing, end-point reflections, and optical nonlinearities. These limitations have motivated the study of light propagation through hollow cores in metallic [27], [28], dielectric [34], microstructured silica [13] and polymer [15], [16], and Bragg waveguides [3], [32], [35]. Hollow-core fibers promise high-energy laser delivery [4], [30], atom or particle guiding [36], [37], chemical and biological molecules sensing [38], and high harmonic generation [39]. Having electrical transmission lines in close contact with an optical counterpart is critical for certain applications [40]. To date, incorporation of optical and electrical functionalities in micrometer-scale fibers has not been achieved. We present the design and fabrication of a new hybrid fiber structure that allows us to transmit electrons and photons simultaneously [5].

The macroscopic preform of hybrid fiber was assembled by wrapping a PEI film (8- $\mu$ m-thick, 20-cm-wide, and 1-m-long), coated on both sides with a 2.6- $\mu$ m-thick layer of $\mathrm{As}_{2} \mathrm{Se}_{3}$, around a pyrex tube having 16-mm outer diameter. An array of Sn strands were positioned around the outer surface of the multilayer structure by using a polymer solution of $20 \%$ PES, $80 \% N, N$-dimethylacetamide. The resulting preform was then consolidated in a vertical rotating furnace at a temperature of $260^{\circ} \mathrm{C}$ and a pressure of $10^{-3}$ torr. The preform longitudinal axis is held vertically in the furnace and a zone-refining heating process is carried out along the preform length. After consolidation, the preform was immersed in a liquid HF bath for $3 \mathrm{~h}$ to selectively etch away the pyrex tube in the center, leaving a hollow core. The finalized preform was then drawn under the following conditions: a temperature of $302{ }^{\circ} \mathrm{C}$, a downfeed speed of $0.003 \mathrm{~mm} / \mathrm{s}$, and a capstan speed of $1 \mathrm{~m} / \mathrm{min}$. The metalcore polymer-cladded fiber strands used in the preform were obtained by drawing a preform with $5-\mathrm{mm}$ Sn (99.75\% purity) core and 7.5-mm PES cladding. The preform was consolidated in a vacuum oven at $260^{\circ} \mathrm{C}$, and then drawn at $-305^{\circ} \mathrm{C}$ in

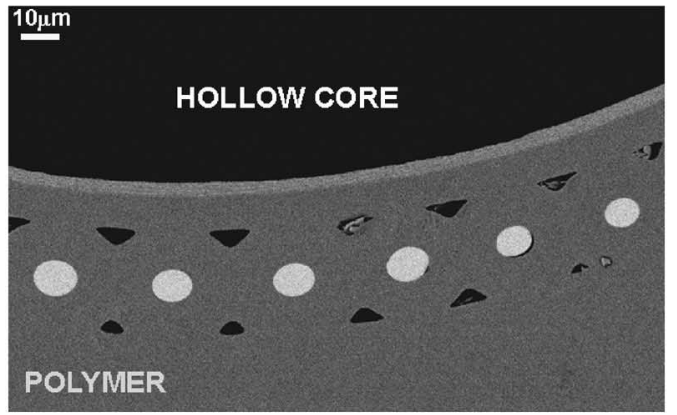

(a)

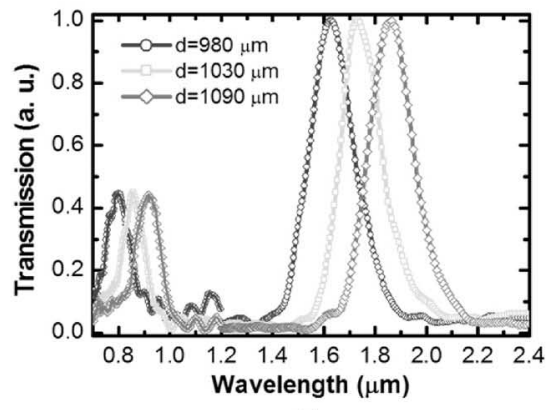

(c)

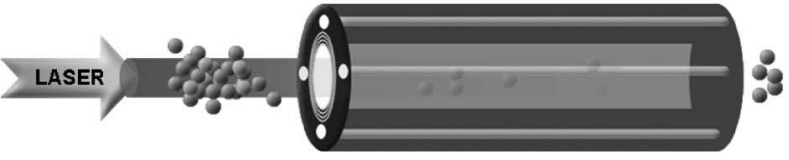

(e)

Fig. 7. Dual electron-photon transport fiber. (a) SEM micrograph of the cross section of the hybrid fiber with a $800-\mu \mathrm{m}$ hollow core, omnidirectional mirror layers, metallic filament array, and polymer cladding. (b) Magnified SEM micrograph of eight pairs of quarter wave dielectric $\mathrm{As}_{2} \mathrm{Se}_{3} / \mathrm{PEI}$ multilayers with submicrometer feature sizes and a metallic microwire. (c) Measured transmission spectra of three fibers having different outer diameters exhibiting PBG guidance through the air core. The primary and the second-order photonic bandgaps are located at 1.62 and $0.8 \mu \mathrm{m}$ for the $980-\mu \mathrm{m}$-thick fiber, and are shifted to longer wavelengths as the fiber diameter increases. (d) Measured electrical current along the $980-\mu \mathrm{m}$-thick, 15 -cm-long fiber, as a function of the applied bias voltage. (e) Schematics of the laser-assisted atom or particle guidance through hollow-core hybrid fibers.

a vertical tube furnace. Both ends of the preform were sealed with polymer to confine the metal during the consolidation and drawing processes. Meters of metallic fibers with outer diameters ranging from $500 \mu \mathrm{m}$ to $1.2 \mathrm{~mm}$ were successfully drawn and sectioned into strands.

The dual electron-photon fiber, shown in Fig. 7(a), is comprised of a hollow core, surrounded by a multilayer dielectric mirror [7], [32] formed from eight pairs of alternating layers of $\mathrm{As}_{2} \mathrm{Se}_{3}$ and PEI (layer thicknesses of 150 and $280 \mathrm{~nm}$, respectively). This multilayer structure provides the optical confinement to the low-index core. Immediately adjacent to this omnidirectional mirror is a circular array of $60 \mathrm{Sn}$ metal strands with diameters of $\sim 8 \mu \mathrm{m}$ each. The whole fiber is then surrounded with a PES polymer cladding.

The optical transport characteristics of the fiber are determined by the positions of the photonic bandgaps. For example, fibers having outer diameters of 980, 1030, and $1090 \mu \mathrm{m}$ have fundamental bandgaps centered at $1.62,1.75$, and $1.85 \mu \mathrm{m}$, and second-order bandgaps around $0.80,0.85$, and $0.92 \mu \mathrm{m}$, 
respectively [see Fig. 7(c)]. Optical transmission spectra were determined using an FTIR spectrometer (Bruker Tensor 37). In order to examine the electrical properties of the fiber, however, both ends of the fiber were coated with a thick layer of gold that electrically connects all the Sn wires. The $I-V$ characteristics of the 980- $\mu \mathrm{m}$-thick fiber are displayed in Fig. 7(d), showing clear ohmic response.

We have performed a systematic investigation of the losses incurred upon propagation through simple (non metal containing) hollow-core multilayer photonic bandgap fibers in [4] and [7]. The loss of such fibers for radiation at a wavelength of $10.6 \mu \mathrm{m}$ was found to be less than $1 \mathrm{~dB} / \mathrm{m}$ [4]. More recently, we have characterized such fibers at a wavelength of $1.5 \mu \mathrm{m}$, and measured the losses to be $2.0 \mathrm{~dB} / \mathrm{m}$. The loss mechanisms are found to be: 1) radiation through the multilayers; 2) intrinsic material absorption; 3) nonuniformity in the layer thicknesses; and 4) scattering due to internal surface roughness. Since light is strongly confined within the hollow core by the cylindrical omnidirectional mirror, it is expected that the presence of additional material components or structural complexity lying outside of the cylindrical mirror will not significantly affect the losses through this hollow fiber. Indeed, initial loss measurements on the electron-photon transport fibers yield loss levels that are comparable to the cylindrical photonic bandgap fibers of similar structural characteristics.

There has been widespread interest in guiding cold atoms, particles, and bacteria in hollow-core optical fibers via radiation pressure [36], [37] or magnetic quadrupole [41]. We expect that the hybrid PBG optical fibers can improve mesoscale particle guidance in miniature transmission lines in many respects [see Fig. 7(e)]: 1) Strong omnidirectional confinement yields much longer transport length due to low loss. 2) Flexibility of polymeric fibers and low-bending loss can enhance guiding efficiency through sharp corners. 3) Generating an electric field by applying potential differences on electrodes allows the separation of charged particles from neutral ones. 4) Generating a quadrupole at the center of fiber can further confine particles in a very small region.

\section{Spectral BroAdening IN Microstructured SOLID GLASS/POLYMER FIBERS}

Spectral broadening of laser pulses via self-phase-modulation (SPM) [42] in single-mode chalcogenide optical fibers has recently attracted much attention for supercontinuum generation [43]-[45]. Since chalcogenide glasses have a high nonlinear index $\left(n_{2}\right)$ compared to silica (two to three orders of magnitude depending on composition), one may obtain significant spectral broadening in very short fibers or even waveguides on a chip. Here, we report on a new microstructured, all-solid, glass/polymer fibers, and investigated the optical and nonlinear properties of these fibers [11].

The small-core fibers were fabricated as follows.

1) 10-mm-thick $\mathrm{As}_{2} \mathrm{Se}_{3}$ rod was synthesized as explained in Section II.

2) A 25-mm-thick cylindrical preform was obtained by rolling $75-\mu \mathrm{m}$-thick PES film onto the rod.

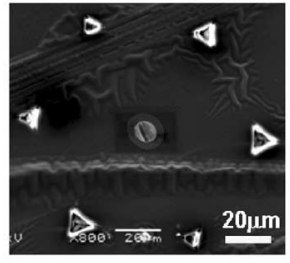

(a)

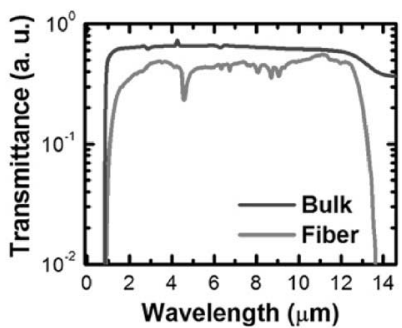

(d)

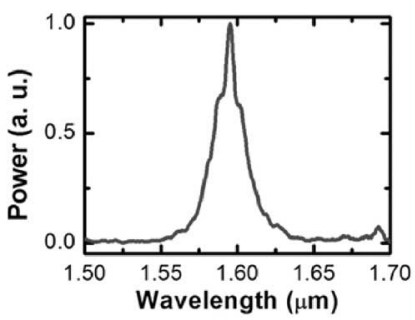

(f)

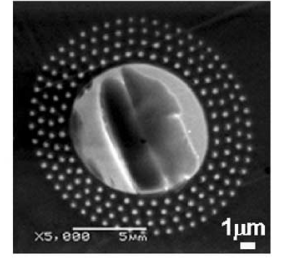

(b)

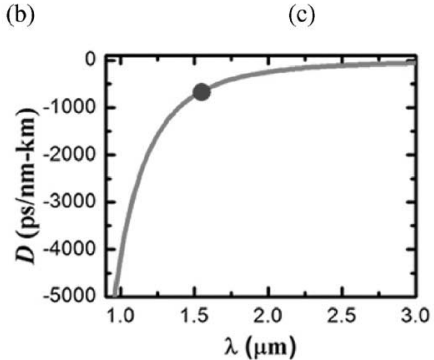

(e)

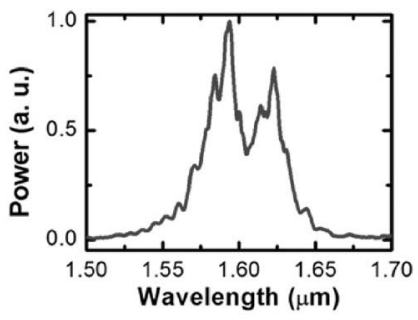

(g)
Fig. 8. Nonlinear, microstructured, all-solid, small-core chalcogenide/ polymer fiber. (a) SEM micrograph of the cross section of a small-core fiber. (b) Magnified SEM shows $9-\mu \mathrm{m}$ glass core $\left(\mathrm{As}_{2} \mathrm{Se}_{3}\right)$ and four rings of glass/polymer nanostructured cladding. (c) Microscopic image of light coming out from a small-core fiber at $1.6-\mu \mathrm{m}$ wavelength. (d) Broadband FTIR transmission spectrum for a bulk-glass disk and a large-core $(300-\mu \mathrm{m})$ fiber. (e) Material dispersion for bulk $\mathrm{As}_{2} \mathrm{Se}_{3}$ glass obtained from ellipsometric measurements. (f) Output spectra for $1.7-\mathrm{mW}$ average input power. (g) Spectral broadening at $56-\mathrm{mW}$ input power.

3) The preform was consolidated in a vacuum oven and subsequently drawn into $750-\mu$ m-thick fibers.

4) Some of these fibers were cut into 20-cm-long pieces, and placed around 9-mm-thick $\mathrm{As}_{2} \mathrm{Se}_{3}$ rod in order to construct four rings.

5) After rolling some PES as a protective cladding; 23-mmthick preform was consolidated and drawn into fibers.

6) A 20 -cm-long and 830- $\mu$ m-thick fiber was placed inside a previously drawn hollow PES fiber (core and outer diameters are around $830 \mu \mathrm{m}$ and $3.1 \mathrm{~mm}$, respectively).

7) A 33-mm-thick preform was obtained by rolling some PES film onto this thick PES fiber, and then consolidated; subsequently drawn into fibers having diameters between 400 and $900 \mu \mathrm{m}$.

Fig. 8(a) shows the SEM micrograph of an 880- $\mu$ m-thick fiber having 9- $\mu$ m-thick $\mathrm{As}_{2} \mathrm{Se}_{3}$ core surrounded by four nanostructured rings [Fig. 8(b)]. A laser beam at $1.6-\mu \mathrm{m}$ wavelength was coupled to a $15-\mathrm{cm}-$ long fiber, and the output beam profile measured by using a high-resolution detector (Sensor Unlimited). As shown in Fig. 8(c), the output beam profile is Gaussian and single mode.

The broadband transmission spectra of a bulk sample (9 $\mathrm{mm}$ in diameter and 3-mm-thick) and a large-core fiber 
(10-cm-long and 300- $\mu \mathrm{m}$-thick core) were measured by using an FTIR (Bruker, Tensor 37). Both sides of the disk and fiber were polished by using $10,3,1$, and $0.3 \mu \mathrm{m}$ aluminum oxide films (South Bay Technology) in order to get optical surfaces. As shown in Fig. 8(d), the bulk $\mathrm{As}_{2} \mathrm{Se}_{3}$ glass has a wide transparency window starting $0.8 \mu \mathrm{m}$ up to $16 \mu \mathrm{m}$ while large-core fiber transmits light between 0.9 and $13.5 \mu \mathrm{m}$. The absorption related to $\mathrm{H}-\mathrm{Se}(\lambda=4.25 \mu \mathrm{m})$ impurities could be removed after further distillation [46].

The refractive index of the bulk $\mathrm{As}_{2} \mathrm{Se}_{3}$ glass was measured by using a spectroscopic ellipsometer (Sopra GES5 UVVis-IR), and fitted to the Cauchy formula: $n(\lambda)=A+B$ / $\lambda^{2}+C / \lambda^{4}$, with parameters $A=2.7391, B=0.0599$, and $C=0.0443$. The material dispersion was determined from $D=-\lambda / c d^{2} n / d \lambda^{2}$, where $c$ is the speed of light. As shown in Fig. 8(e), the dispersion is negative and large around the telecommunications wavelength, and gets smaller for longer wavelengths. We estimated $D=-552 \mathrm{ps} / \mathrm{nm}-\mathrm{km}$ at $1.55 \mu \mathrm{m}$, which is very close to the value reported in [43].

In order to demonstrate the spectral broadening via SPM in our small-core microstructured fibers, we coupled an OPO laser (Mira, Coherent) beam by using a $\times 20$ microscope objective to 3 -cm-long and $6.3-\mu \mathrm{m}$-core fiber. The output light was collected with $\times 100$ microscope objective and sent to an optical spectrum analyzer (Ando) via a multimode silica fiber. While the spectrum is identical with input spectrum for low power $(1.7 \mathrm{~mW})$, there is a significant spectral broadening at high powers. We observed a spectrum broadening of about $150 \%$ for $56-\mathrm{mW}$ input power compared to the input spectrum [Fig. 8(g)]. Modification of the nanostructure around the solid core allows tailoring the total dispersion.

\section{CONCLUSION}

We have introduced multimaterial composite fibers and fiberbased devices having optical, electrical, and thermal functionalities at nanometer feature sizes and kilometer-length scales, enabled by innovative fabrication approaches that allows for the use of new materials and geometries that have not been, hitherto, associated with fibers. By combining materials and exploring novel geometries, conventional functionalities can be enhanced and new ones introduced. Thus, a large repertoire of structures and devices with electronic, photonic, and thermal properties by design may indeed be processed by the familiar thermal drawing of macroscopic preforms.

\section{ACKNOWLEDGMENT}

The authors would like to thank N. Orf, G. Benoit, S. D. Hart, K. Kuriki, Y. Kuriki, E. E. Bayindir, and P. H. Prideaux for their help during various stages of this paper.

\section{REFERENCES}

[1] S. L. Chuang, Physics of Optoelectronic Devices. New York: Wiley, 1995.

[2] S. Donati, Photodetectors: Devices, Circuits, and Applications. Englewood Cliffs, NJ: Prentice-Hall, 2000.
[3] S. D. Hart, G. R. Maskaly, B. Temelkuran, P. H. Prideaux, J. D. Joannopoulos, and Y. Fink, "External reflection from omnidirectional dielectric mirror fibers," Science, vol. 296, pp. 510-513, Apr. 2002

[4] B. Temelkuran, S. D. Hart, G. Benoit, J. D. Joannopoulos, and Y. Fink, "Wavelength-scalable hollow optical fibres with large photonic bandgaps for $\mathrm{CO}_{2}$ laser transmission," Nature, vol. 420, pp. 650-653, Dec. 2002.

[5] M. Bayindir, F. Sorin, A. F. Abouraddy, J. Viens, S. D. Hart, J. D. Joannopoulos, and Y. Fink, "Metal-insulator-semiconductor optoelectronic fibres," Nature, vol. 431, pp. 826-829, Oct. 2004.

[6] M. Bayindir, A. F. Abouraddy, F. Sorin, J. D. Joannopoulos, and Y. Fink, "Fiber photodetectors codrawn from conducting, semiconducting, and insulating materials," Opt. Photonics News, vol. 15, p. 24, Dec. 2004.

[7] K. Kuriki, O. Shapira, S. D. Hart, G. Benoit, Y. Kuriki, J. F. Viens, M. Bayindir, J. D. Joannopoulos, and Y. Fink, "Hollow multilayer photonic bandgap fibers for NIR applications," Opt. Express, vol. 12, pp. 1510 1517, Apr. 2004

[8] M. Bayindir, O. Shapira, D. Saygin-Hinczewski, J. Viens, A. F. Abouraddy, J. D. Joannopoulos, and Y. Fink, "Integrated fibres for self-monitored optical transport," Nat. Mater., vol. 4, pp. 820-825, Nov. 2005.

[9] M. Bayindir, A. F. Abouraddy, J. Arnold, J. D. Joannopoulos, and Y. Fink, "Thermal-sensing mesoscopic fiber devices by composite-material processing," Adv. Mater., vol. 18, pp. 845-849, Apr. 2006.

[10] A. F. Abouraddy, O. Shapira, M. Bayindir, J. Arnold, F. Sorin, D. S. Hinczewski, J. D. Joannopoulos, and Y. Fink, "Large-area opticalfield measurements with geometric fibre constructs," Nat. Mater., vol. 5, pp. 532-536, 2006.

[11] M. Bayindir, A. F. Abouraddy, J. D. Joannopoulos, and Y. Fink, "Supercontinuum generation in solid chalcogenide glass/polymer fibers," to be published.

[12] P. Russell, "Photonic crystal fibers," Science, vol. 299, pp. 358-362, Jan. 2003.

[13] J. C. Knight, "Photonic crystal fibres," Nature, vol. 424, pp. 847-851, Aug. 2003.

[14] B. J. Eggleton, C. Kerbage, P. Westbrook, R. S. Windeler, and A. Hale, "Microstructured optical fiber devices," Opt. Express, vol. 9, pp. 698-713, Dec. 2001.

[15] M. van Eijkelenborg, M. Large, A. Argyros, J. Zagari, S. Manos, N. A. Issa, I. M. Bassett, S. C. Fleming, R. C. McPhedran, C. M. de Sterke, and N. A. P. Nicorovici, "Microstructured polymer optical fibre," Opt. Express, vol. 9, pp. 319-327, Sep. 2001.

[16] A. Argyros, M. A. van Eijkelenborg, M. C. Large, and I. M. Bassett, "Hollow-core microstructured polymer optical fiber," Opt. Lett., vol. 31, pp. 172-174, Jan. 2006.

[17] S. D. Hart, "Multilayer composite photonic bandgap fibers" Ph.D. dissertation, Massachusetts Inst. Technol., Cambridge, MA, 2004

[18] M. A. Popescu, Non-Crystalline Chalcogenides. Dortrecht, The Netherlands: Kluwer, 2000.

[19] Z. U. Borisova, Glassy Semiconductors. New York: Plenum, 1984.

[20] J. M. Harbold, F. Ilday, F. W. Wise, J. S. Sanghera, V. Q. Nguyen, L. B. Shaw, and I. D. Aggarwal, "Highly nonlinear As-S-Se glasses for all-optical switching," Opt. Lett., vol. 27, pp. 119-121, Jan. 2002.

[21] G. Benoit and Y. Fink. Database of optical constants. [Online] Available: http://mit-pbg.mit.edu/Pages /Ellipsometry.html

[22] W. A. King, A. G. Clare, and W. C. Lacourse, "Laboratory preparation of highly pure $\mathrm{As}_{2} \mathrm{Se}_{3}$ glass," J. Non-Cryst. Solids, vol. 181, pp. 231-237, Feb. 1995.

[23] I. Inagawa, R. Iizuka, T. Yamagishi, and R. Yokota, "Optical and thermal properties of chalcogenide Ge-As-Se-Te glasses for IR fibers," J. NonCryst. Solids, vol. 95-96, pp. 801-808, Dec. 1987.

[24] V. Q. Nguyen, J. S. Sanghera, F. H. Kung, P. C. Pureza, and I. D. Aggarwal, "Very large temperature-induced absorptive loss in high Te-containing chalcogenide fibers," J. Lightw. Technol., vol. 18, pp. 1395-1401, Oct. 2000.

[25] V. K. Tikhomirov, D. Furniss, A. B. Seddon, J. A. Savage, P. D. Mason, D. A. Orchard, and K. L. Lewis, "Glass formation in the Te-enriched part of the quaternary $\mathrm{Ge}-\mathrm{As}-\mathrm{Se}-\mathrm{Te}$ system and its implication for midinfrared optical fibres," Infrared Phys. Technol., vol. 45, pp. 115-123, Mar. 2004.

[26] G. Benoit, K. Kuriki, J. F. Viens, J. D. Joannopoulos, and Y. Fink, "Dynamic all-optical tuning of transverse resonant cavity modes in photonic bandgap fibers," Opt. Lett., vol. 30, pp. 1620-1622, Jul. 2004.

[27] T. Abel, J. Hirsch, and J. A. Harrington, "Hollow glass waveguides for broadband infrared transmission," Opt. Lett., vol. 19, pp. 1034-1036, Jul. 1994. 
[28] T. Katagiri, Y. Matsuura, and M. Miyagi, "Metal-covered photonic bandgap multilayer for infrared hollow waveguides," Appl. Opt., vol. 41, pp. 7603-7606, Dec. 2002.

[29] A. Dayan, A. Goren, and I. Gannot, "Theoretical and experimental investigation of the thermal effects within body cavities during transendoscopical $\mathrm{CO}_{2}$ laser-based surgery," Laser Surg. Med., vol. 35, pp. 18-27, Jul. 2004.

[30] J. A. Harrington, Infrared Fibers and Their Applications. Bellingham, WA: SPIE, 2004

[31] A. K. Devaiah, S. M. Shapshay, U. Desai, G. Shapira, O. Weisberg, D. S. Torres, and Z. Wang, "Surgical utility of a new carbon dioxide laser fiber: Functional and histological study," Laryngoscope, vol. 115, pp. 1463-1468, Aug. 2005.

[32] Y. Fink, J. N. Winn, S. Fan, C. Chen, J. Michel, J. D. Joannopoulos, and E. L. Thomas, "A dielectric omnidirectional reflector," Science, vol. 282, pp. 1679-1682, Nov. 1998.

[33] M. Bayindir, J. D. Joannopoulos, and Y. Fink, "Fiber-based tunable attenuators for infrared wavelengths," unpublished.

[34] E. A. J. Marcatili and R. A. Schmeltzer, "Hollow metallic and dielectric wave-guides for long distance optical transmission and lasers," Bell Syst. Tech. J., pp. 1783-1809, Jul. 1964.

[35] P. Yeh, A. Yariv, and E. Marom, "Theory of Bragg fiber," J. Opt. Soc. Amer., vol. 68, pp. 1196-1201, Sep. 1978.

[36] M. J. Renn, D. Montgomery, O. Vdovin, D. Z. Anderson, C. E. Wieman, and E. A. Cornell, "Laser-guided atoms in hollow-core optical fibers," Phys. Rev. Lett., vol. 75, pp. 3253-3256, Oct. 1995.

[37] F. Benabid, J. C. Knight, and P. S. J. Russell, "Particle levitation and guidance in hollow-core photonic crystal fiber," Opt. Express, vol. 10, pp. 1195-1203, Oct. 2002.

[38] C. Charlton, B. Temelkuran, G. Dellemann, and B. Mizaikoff, "Midinfrared sensors meet nanotechnology: Trace gas sensing with quantum cascade lasers inside photonic band-gap hollow waveguides," Appl. Phys. Lett., vol. 86, no. 194102, May 2005.

[39] A. Rundquist, C. G. Durfee, Z. Chang, C. Herne, S. Backus, M. M. Murnane, and H. C. Kapteyn, "Phase-matched generation of coherent soft X-rays," Science, vol. 280, pp. 1412-1415, May 1998.

[40] M. Large, A. Argyros, G. Barton, I. Bassett, F. Cox, M. Fellew, G. Henry, N. Issa, S. Manos, W. Padden, L. Poladian, M. A. van Eijkelenborg, and J. Zagari, "Microstructured optical fibers: Why use polymers?," in Proc. 29th ECOC'03, Rimini, Italy, pp. 1014-1017.

[41] M. Key, I. G. Hughes, W. Rooijakkers, B. E. Sauer, E. A. Hinds, D. J. Richardson, and P. G. Kazansky, "Propagation of cold atoms along a miniature magnetic guide," Phys. Rev. Lett., vol. 84, pp. 1371-1373, Feb. 2000.

[42] R. H. Stolen and C. Lin, "Self-phase-modulation in silica optical fibers," Phys. Rev. A, vol. 17, pp. 1448-1453, Apr. 1978

[43] R. E. Slusher, G. Lenz, J. Hodelin, J. Sanghera, L. B. Shaw, and I. D. Aggarwal, "Large Raman gain and nonlinear phase shifts in highpurity $\mathrm{As}_{2} \mathrm{Se}_{3}$ chalcogenide fibers," J. Opt. Soc. Amer. B, Opt. Phys., vol. 21, pp. 1146-1155, Jun. 2004.

[44] J. T. Gopinath, H. M. Shen, H. Sotobayashi, E. P. Ippen, T. Hasegawa, T. Nagashima, and N. Sugimoto, "Highly nonlinear bismuth-oxide fiber for smooth supercontinuum generation at $1.5 \mu \mathrm{m}$," Opt. Express, vol. 12 pp. 5697-5702, Nov. 2004.

[45] L. B. Fu, M. Rochette, V. G. Ta'eed, D. J. Moss, and B. J. Eggleton, "Investigation of self-phase modulation based optical regeneration in single mode $\mathrm{As}_{2} \mathrm{Se}_{3}$ chalcogenide glass fiber," Opt. Express, vol. 13, pp. 7637 7644, Jan. 2005.

[46] V. Q. Nguyen, J. S. Sanghera, P. C. Pureza, F. H. Kung, and I. D. Aggarwal, "Fabrication of arsenic selenide optical fiber with low hydrogen impurities," J. Amer. Ceram. Soc., vol. 85, pp. 2849-2851, Nov. 2002.

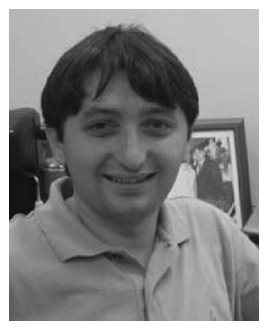

Mehmet Bayindir received the B.S., M.S., and Ph.D. degrees, all in physics, from Bilkent University, Ankara, Turkey, in 1995, 1997, and 2002, respectively.

He was a Research Scientist with the Massachusetts Institute of Technology, Cambridge, where he was engaged in the design, fabrication, and characterization of various types of fibers, made of conducting, semiconducting, and insulating materials that are in close contact and in a variety of geometries, paving the way for the future development of fibers and woven fabrics with novel optical and electrical properties. Currently, he is an Assistant Professor in the Department of Physics, Bilkent University. He is the author or coauthor of over 35 journal papers, more than 25 refereed conference papers, and a book (in Turkish). He holds five U.S. patents. His research interests include several problems in theoretical condensed physics such as impurity effects in high-temperature superconductivity, localization in quantum Hall systems, persistent current in mesoscopic rings, and Bose-Einstein condensation in low-dimensional systems.

Dr. Bayindir is a Reviewer for several scientific journals including Physical Review Letters, Optics Letters, Physical Review B, Physical Review E, Optics Express, Journal of the Optical Society of America B, Physics Letters A, and several IEEE journals.

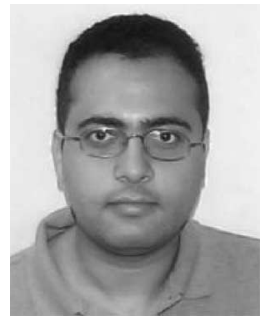

Ayman F. Abouraddy received the B.S. and M.S. degrees from Alexandria University, Alexandria, Egypt, in 1994 and 1997, respectively, and the Ph.D. degree from Boston University, Boston, MA, in 2003, all in electrical engineering.

Since 2003, he has been with the Massachusetts Institute of Technology, Cambridge, where he is currently a Research Scientist at the Research Laboratory of Electronics, pursuing research on novel optical fiber structures, nanophotonics, fiber-based optoelectronic devices, and optical imaging using large-scale three-dimensional arrays constructed from photosensitive fibers. He is also engaged in investigating techniques that lead to sub-diffraction limited resolution in optical microscopy and lithography. He is the coauthor of almost 30 journal publications, and holds three patents. His research has been concerned with optical physics, quantum optics, and quantum information theory. He has also investigated nonlinear optical phenomena in bulk materials and optical fibers. His current research interests include the Einstein-Podolsky-Rosen paradox, quantum nonlocality, and quantum cryptography.

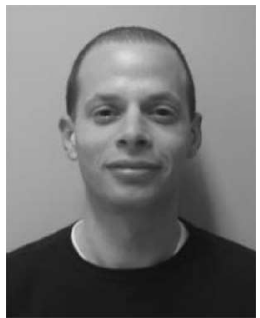

Ofer Shapira received the B.Sc. and M.Sc. degrees, both in electrical engineering, from the Technion, Haifa, Israel, in 1997 and 1999, respectively. He is currently working toward the Ph.D. degree at the Massachusetts Institute of Technology, Cambridge.

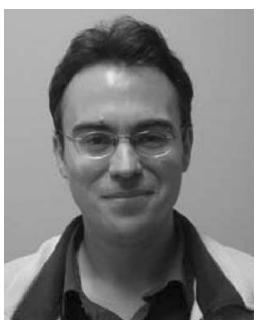

Jeff Viens received the B.Sc.A. and M.S. degrees from Laval University, Quebec City, QC, Canada, in 1995 and 1997, respectively. He is currently working toward the Ph.D. degree at the Massachusetts Institute of Technology, Cambridge.

He possesses seven years of engineering experience in research and industrial settings. He is the coauthor of 21 papers and holds nine U.S. patents. His research interests include the design and fabrication of multimaterial fibers.

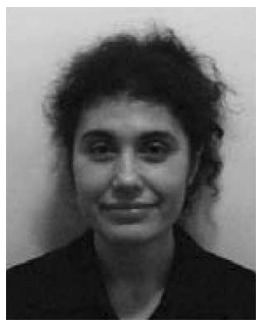

Dursen Saygin-Hinczewski received the B.S. and M.S. degrees, both in physics, from Istanbul Technical University, Istanbul, Turkey, in 1997 and 2000, respectively, where she is currently working toward the Ph.D. degree.

During 2004 and 2005, she was a Visiting Student in the Photonic Bandgap Fibers and Devices Group at the Massachusetts Institute of Technology, Cambridge, where she was engaged in studying the role of surface energy and thermal-drawing conditions on the fabrication of metal-containing photonic bandgap fibers. Her research is based on optical filters for different wavelength regions, electrochromic devices, and gas/humidity sensors derived by sol-gel spin or dip-coating processes. 


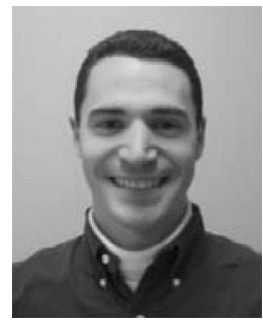

Fabien Sorin received the B.S. and M.S. degrees, both in physics, from Ecole Polytechnique, Palaiseau, France, in 2001 and 2002, respectively.

He is currently a Graduate Student and a Research Associate in the Photonic Bandgap Fibers and Devices Group, Massachusetts Institute of Technology, Cambridge. His research interests include the design, fabrication, and characterization of optoelectronic hybrid fiber devices for applications in large area optoelectronic screens and smart fabrics.

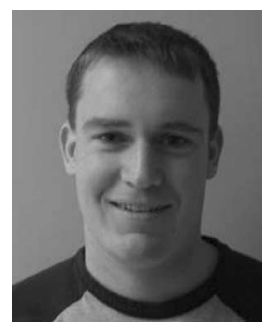

Jerimy Arnold received the B.S. degree in electrical engineering and the M.E. degree in electrical engineering and computer science from the Massachusetts Institute of Technology, Cambridge, in 2004 and 2005, respectively.

$\mathrm{He}$ is currently a Research Associate in the Photonic Bandgap Fibers and Devices Group, Massachusetts Institute of Technology. His research interests include the design and implementation of optical detector array systems derived from metal-insulatorsemiconductor optoelectronic fibers.

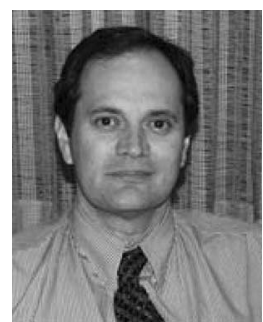

John D. Joannopoulos received the B.A. and Ph.D. degrees, both in physics, from the University of California, Berkeley, in 1968 and 1974, respectively.

Since 1974, he has been at the Massachusetts Institute of Technology, Cambridge, where he is currently the Francis Wright Davis Professor of Physics and the Director of the Institute for Soldier Nanotechnologies. He is the author or coauthor of more than 440 journal articles, two textbooks on photonic crystals, and holds 25 U.S. patents.

Dr. Joannopoulos is a member of the American Association for the Advancement of Science and the Materials Research Society. He was both an Alfred P. Sloan Fellow and a John S. Guggenheim Fellow of the American Physical Society. He was a Divisional Associate Editor of Physical Review Letters, and was on the Editorial Board of Reviews of Modern Physics. He was the recipient of the MIT School of Science Graduate Teaching Award in 1991, the William Buechner Teaching Prize of the Department of Physics in 1996, and the David Adler Award of the American Physical Society in 1997.

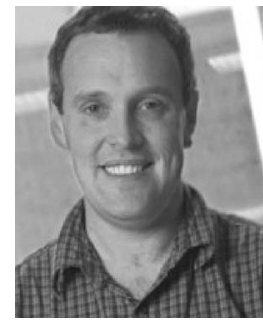

Yoel Fink received the B.Sc. degree in chemical engineering and the B.A. degree in physics from the Technion, Haifa, Israel, in 1994 and 1995, respectively, and the Ph.D. degree from the Massachusetts Institute of Technology, Cambridge, in 2000.

Since 2005, he has been the Thomas B. King Associate Professor of Materials Science in the Department of Materials Science and Engineering, Massachusetts Institute of Technology. In 2000, he cofounded OmniGuide Inc., and is currently on its Board of Directors. He is the coauthor of more than 35 journal articles and holds 18 U.S. patents on photonic fibers and devices. His current research interests include the design, fabrication, and characterization of solid-state mesostructured fibers containing metals, insulators, and semiconducting materials for optical, electronic, and thermal applications.

Dr. Fink was a recipient of the National Amos De-Shalit Foundation Scholarship, in 1992, was the winner of the Hershel Rich Technion Innovation Competition, in 1994, was the recipient of the Technology Review Award for the 100 Top Young Innovators, in 1999, and the National Academy of Sciences Initiatives in Research Award in 2004 